\title{
Remote ischaemic preconditioning in coronary artery bypass surgery: a meta-analysis
}

\author{
Fabrizio D’Ascenzo, ${ }^{1,2}$ Erika Cavallero, ${ }^{1}$ Claudio Moretti, ${ }^{1,2}$ Pierluigi Omedè, \\ Filippo Sciuto, ${ }^{1}$ Ishtiaq A Rahman, ${ }^{3}$ Robert S Bonser, ${ }^{3}$ Jeon Yunseok, ${ }^{4}$ \\ Robert Wagner, ${ }^{5}$ Tomas Freiberger ${ }^{5,6}$ Gudrun Kunst, ${ }^{7}$ Michael S Marber, ${ }^{7}$ \\ Matthias Thielmann, ${ }^{8}$ Bingyang $\mathrm{Ji}^{9}{ }^{9}$ Yasser M Amr, ${ }^{10}$ Maria Grazia Modena, ${ }^{11}$ \\ Giuseppe Biondi Zoccai, ${ }^{2,11}$ Imad Sheiban, ${ }^{1}$ Fiorenzo Gaita ${ }^{1}$
}

- Additional materials are published online only. To view these files please visit the journal online (http://heart.bmj. com/content/98/17.toc).

1 Division of Cardiology, University of Turin, Turin, Italy ${ }^{2}$ Meta-analysis and Evidence based medicine Training in Cardiology (METCARDIO),

Ospedaletti, Italy

${ }^{3}$ Department of Cardiothoracic Surgery University Hospital Birmingham NHS Trust,

Birmingham, UK

${ }^{4}$ Department of Anaesthesiology and Pain Medicine, Seoul

National University Hospital, Seoul, Korea

${ }^{5}$ Centre for Cardiovascular Surgery and Transplantation Brno, Czech Republic ${ }^{6}$ Ceitec, Masaryk University, Brno, Czech Republic ${ }^{7}$ Department of Anaesthetics, Intensive Care Medicine and Pain Therapy, King's College Hospital NHS Foundation Trust, London, UK

${ }^{8}$ Department of Thoracic and Cardiovascular Surgery, West-German Heart Center Essen, University Hospital Essen, Germany

${ }^{9}$ Department of

Cardiopulmonary Bypass,

Cardiovascular Institute \& Fuwai Hospital, PUMC \& CAMS, Beiijing, China

${ }^{10}$ Tanta faculty of Medicine, Egypt

${ }^{11}$ Division of Cardiology, University of Modena and Reggio Emilia, Modena, Italy

\section{Correspondence to}

Dr Claudio Moretti, Division of Cardiology, University of Turin, Turin, Italy; drclamore@yahoo.it

Received 28 December 2011 Revised 27 February 2012 Accepted 1 April 2012

\begin{abstract}
Aim Randomised trials exploring remote ischaemic preconditioning (RIPC) in patients undergoing coronary artery bypass graft (CABG) surgery have yielded conflicting data regarding potential cardiovascular and renal protection, and are individually flawed by small sample size.
\end{abstract}

Methods Three investigators independently searched the MEDLINE, EMBASE and Cochrane databases to identify randomised trials testing RIPC in patients undergoing $\mathrm{CABG}$.

Results Nine studies with 704 patients were included. Standardised mean difference of troponin I and $T$ release showed a significant decrease $1-0.36(95 \% \mathrm{Cl}-0.62$ to -0.09)). This difference held true after excluding the trials with cross-clamp fibrillation, the study with offpump CABG and studies using a flurane as anaesthetic agent $(-0.41(95 \% \mathrm{Cl}-0.69$ to -0.12$),-0.38(95 \% \mathrm{Cl}$ -0.70 to -0.07$)$ and $-0.37(95 \% \mathrm{Cl}-0.63$ to -0.12$)$, respectively). A similar trend was also obtained for patients with multivessel disease $(-0.41(95 \% \mathrm{Cl}-0.73$ to -0.08$))$. The trials evaluating postoperative creatinine reported a non-significant reduction $10.02195 \% \mathrm{Cl}-0.09$ to 0.13$)$ ). Moreover, the length of in-hospital stay was not influenced by the kind of treatment (weighted mean difference $0.27(95 \% \mathrm{Cl}-0.24$ to 0.79$)$ ).

Conclusion RIPC reduced the release of troponin in patients undergoing CABG. Larger randomised trials are needed to clarify the presence of a causal relationship between RIPC-induced troponin release and clinical adverse events.

\section{INTRODUCTION}

Remote ischaemic preconditioning (RIPC) consists of protection of an organ from a sustained ischaemic event obtained through the application of brief sublethal ischaemia to another tissue. Recently, this gained relevance in scientific debate, thanks to its practical feasibility, limited costs and potential benefits.

First discovered in animal models, ${ }^{1}$ it has been rapidly translated to clinical arenas in different settings such as, congenital heart defect corrections, ${ }^{2}$ abdominal aneurysm repair ${ }^{3}$ and coronary artery percutaneous or surgical revascularisation. ${ }^{45}$ This last context may be the most promising in terms of new strategies for myocardial protection, since the risk profile of patients referred for cardiac surgery remains challenging, and established evidence relates perioperative myocardial injury to postoperative morbidity and mortality. ${ }^{6}$

Unfortunately, available data obtained from randomised controlled trials (RCTs) on RIPC in patients undergoing coronary artery bypass graft (CABG) surgery are inconclusive. Some studies outlined the efficacy of transient upper limb ischaemic preconditioning to reduce serum troponins' release after surgery, ${ }^{4}$ while similar protocols failed to achieve statistically significant differences. As discussed by Rahman et al, ${ }^{8}$ some studies may be underpowered to exclude a lack of protection by RIPC.

In the present review, we thus aimed to conduct a comprehensive evaluation of current data to further guide larger RIPC studies and future implementation of new treatment strategies to prevent myocardial damage or dysfunction in patients undergoing CABG surgery.

\section{METHODS}

\section{Data sources}

The MEDLINE, EMBASE and Cochrane databases were searched using terms such as ischaemic preconditioning' or 'remote ischaemic preconditioning', 'surgical coronary revascularization' or 'coronary artery bypass graft surgery (CABG)', according to optimal search strategies. ${ }^{9}$

Reference lists of articles included were also reviewed, and no language restriction was imposed. All corresponding authors of shortlisted studies were directly contacted for additional data, invited to participate in data analysis and interpretation, and were requested for suggestions for additional studies.

\section{Study selection}

RCTs comparing troponin release after CABG of patients assigned to RIPC or not were included, while those comparing non-RIPC were excluded.

The outcomes of interest were release of cardiac troponin I and $\mathrm{T}$ (defined as area under the curve, or when available, data at 6, 24, $48 \mathrm{~h}$ and at peak), values of creatinine (the highest creatinine valued during follow-up; $\mathrm{mg} / \mathrm{dl}$ ) and length of in-hospital stay.

Three investigators (GBZ, EC, FDA) independently reviewed titles, abstracts and the full texts as needed to determine whether studies met inclusion criteria. Conflicts between reviewers were resolved through re-review and discussion. 


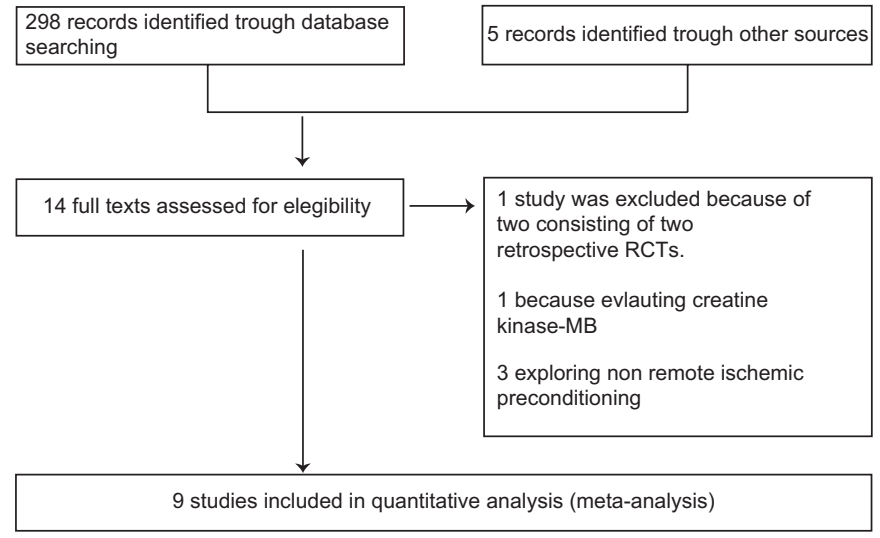

Figure 1 Review profile.

\section{Data extraction and quality assessment}

Three authors (GBZ, EC, FDA) independently abstracted data on study design, setting, RIPC protocols, assay of troponin used and its upper limit of normality, number of patients with multivessel disease (ie, three or more vessels protected with graft), protocols of cardioplegia used, bypass and cross-clamp time.

Quality of included trials was explored according to Cochrane, PRISMA and QUORUM statements; ${ }^{10}{ }^{11}$ methods to obtain sample size, selection bias (allocation and random sequence generation), performance bias (blinding of participants and personnel), detection bias (blinding of outcome assessment) and attrition bias (incomplete outcome data) were assessed and graphically described. Moreover, the Jadad Scale ${ }^{12}$ was also used to appraise methodological quality of included studies.

\section{Data synthesis and analysis}

Random-effects models were exploited to compute standard weighted mean difference (wmd) for troponin $\mathrm{T}$ and I separately for creatinine and for length of in-hospital stay. Fixed-effects models were also tested and their results reported only if different from random effects. Troponin I values were analysed for patients with multivessel disease only; standardised mean difference (smd) was used to evaluate troponin release globally: RevMan 5 (The Cochrane Collaboration, The Nordic Cochrane Centre, Copenhagen, Denmark) was used. Hypothesis testing for statistical homogeneity was set at the two-tailed 0.10 level and based on the Cochran $\mathrm{Q}$ test, with $\mathrm{I}^{2}$ values of $25 \%, 50 \%$ and $75 \%$ representing mild, moderate and extensive statistical heterogeneity, respectively. Funnel plot was explored to identify small-study bias.

\section{RESULTS}

Three hundred and three results were first appraised at abstract level and 14 at full text level (figure 1); one was excluded because

Table 1 Main features of patients

\begin{tabular}{lclll}
\hline Study & $\begin{array}{l}\text { No. of } \\
\text { patients }\end{array}$ & Age (years) & $\begin{array}{l}\text { Multivessel } \\
\text { disease (\%) }\end{array}$ & $\begin{array}{l}\text { Ejection } \\
\text { fraction (\%) }\end{array}$ \\
\hline Hausenloy, 2007 & 57 & 67 & 83 & 53 \\
Hong, 2010 & 130 & 65 & - & 56 \\
Lucchinetti, 2012 & 55 & 60 & 70 & 50 \\
Karuppasamy, 2011 & 54 & 67 & 70 & 50 \\
Kottenberg, 2012 & 72 & 65 & 100 & - \\
Rahman, 2010 & 162 & 63 & 100 & - \\
Thielman, 2010 & 53 & 64 & 100 & 57 \\
Venugopal, 2010 & 55 & 64 & 60 & 51 \\
Wagner, 2010 & 66 & 67 & 80 & 53 \\
\hline
\end{tabular}

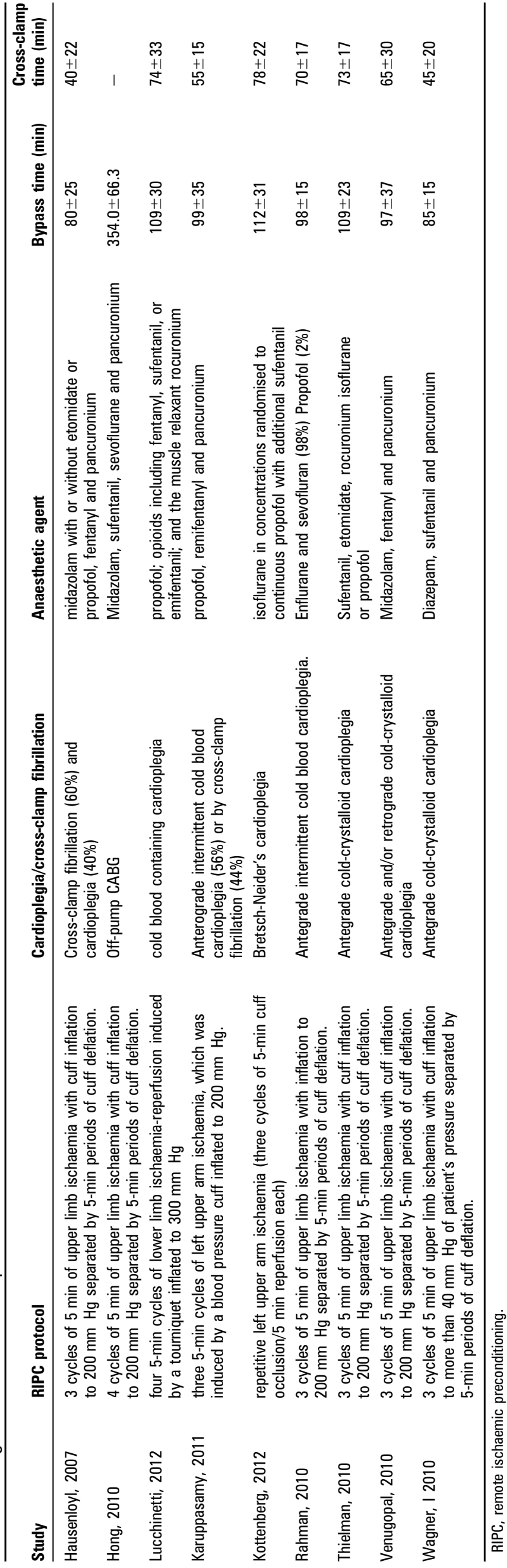




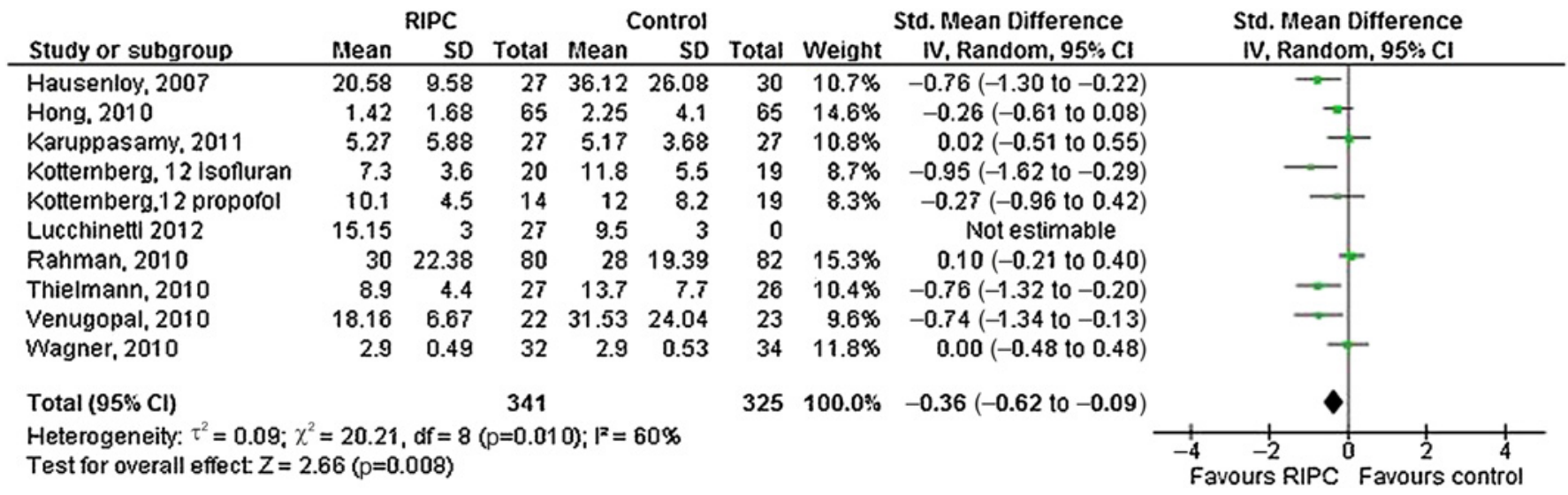

Figure 2 Standardised mean difference of troponin values. RIPC, remote ischaemic preconditioning.

it consisted of a retrospective analysis of two RCTs; ${ }^{13}$ one because it evaluated only creatinine kinase isoenzyme $\mathrm{MB} ;{ }^{14}$ and three because it explored non-RIPC. ${ }^{15-17}$ Finally, $9^{5} 818-25$ RCTs were included.

From a methodological point of view (see online supplementary figure B), sample size was obtained to gain $90 \%$ power and $5 \%$ o in four of the studies; randomisation was generated from computers in four of them, while in three of them, the allocation was performed in numbered containers. Moreover, in four studies, patients, surgeons and doctors evaluating outcomes were blinded; attrition bias was present in two of the six studies. The Jadad scale (see online supplementary table A) showed similar results.

Seven hundred and four patients were included (tables 1 and 2); most of them presented a multivessel disease, without an important impairment of ejection fraction. RIPC protocol was analogous in all studies consisting of three cycles of $5 \mathrm{~min}$ of upper limb ischaemia with inflation to $200 \mathrm{~mm} \mathrm{Hg}$ separated by 5 -min periods of cuff deflation, except for one study in which four cycles were performed.

Smd of troponin I and $\mathrm{T}$ release showed a significant small decrease $(-0.36(95 \%$ CI -0.62 to -0.09$)$ (figure 2$)$. This difference held true after excluding the trials with cross-clamp fibrillation, the study with off-pump CABG and studies using a flurane as anaesthetic agent $(-0.41(95 \% \mathrm{CI}-0.69$ to -0.12$)$ ), $-0.38(95 \%$ CI -0.70 to -0.07$)$ and -0.37 (95\% CI -0.63 to -0.12$)$, respectively). A similar trend was also obtained for patients with only multivessel disease $(-0.41$ (95\% CI -0.73 to -0.08$)$ ). (figure 3)

The trials evaluating postoperative creatinine reported a nonsignificant reduction (0.02 (95\% CI -0.09 to 0.13$)$ ). Moreover, the length of in-hospital stay was not influenced by the kind of treatment (wmd; 0.27 (95\% CI -0.24 to 0.79 )) (figures 4 and 5).

\section{DISCUSSION}

The present article represents the first attempt to perform a pooled analysis about RIPC in CABG. RIPC reduced the amount of cardiac enzyme release in patients undergoing CAGB, while no differences in length of in-hospital stay and creatinine values were reported.

Reduction in troponin release was demonstrated among studies assessing both troponin $\mathrm{T}$ and $\mathrm{I}$, and for patients with surgical revascularisation of single vessel or with multivessel disease. The restoration of blood supply to organs after a certain period of no-flow ischaemia results in parenchymal damage referred to as ischaemia-reperfusion injury.

In an attempt to shed light on the mechanisms underlying cardioprotection, bench and bedside research focused on its physiological substrate. The exact nature of signal transduction from remote tissue to target organ remains to be elucidated. However, three possible main mechanisms have been suggested, which could variably contribute to the phenomena interacting with each other and, therefore, not being mutually exclusive. The abrogation of remotely induced cardioprotection with the ganglion blocker hexamethonium, or the pre-treatment of sensory nerves with capsaicin, suggested a neuronal pathway. ${ }^{26} 27$ However, Konstantinov et $a l^{28}$ demonstrated that RIPC can also protect the denervated transplanted heart, suggesting a humoural hypothesis. Several endogenous molecules have been implicated (opioids, insulin, bradykinin, adenosine). These mediators generated in the organs remote from the heart enter the blood stream and activate specific myocardial receptors that eventually recruit intracellular pathways of cardioprotection. ${ }^{28}$ According to Battipaglia et $a l^{29}$ besides the induction of changes in cardiomyocytes that make them more resistant to ischaemia, RIPC may exert its benefits through an additional humoural mechanism resulting in a reduced platelet reactivity and thus, decreased thrombogenic

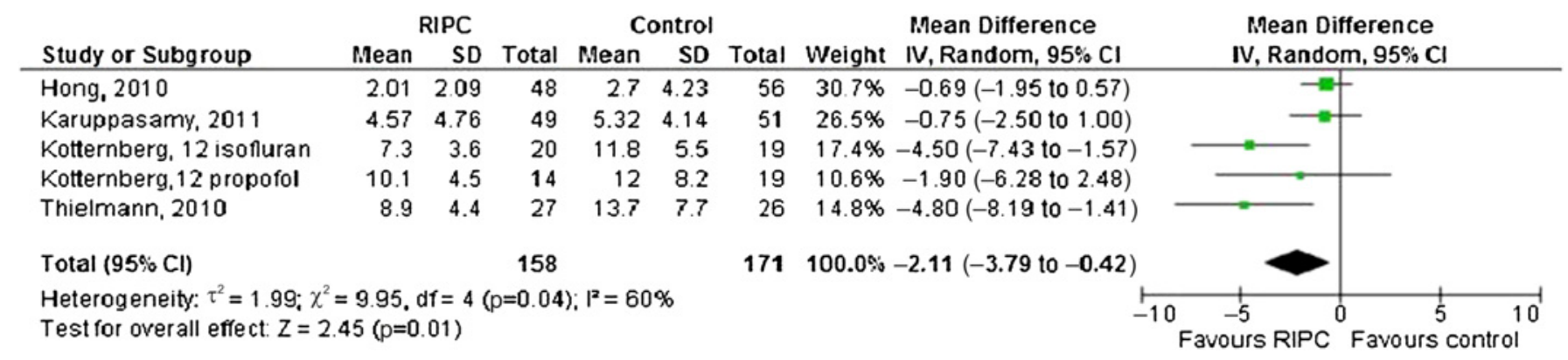

Figure 3 Standardised mean difference of troponin values in patients with multivessel disease. RIPC, remote ischaemic preconditioning. 


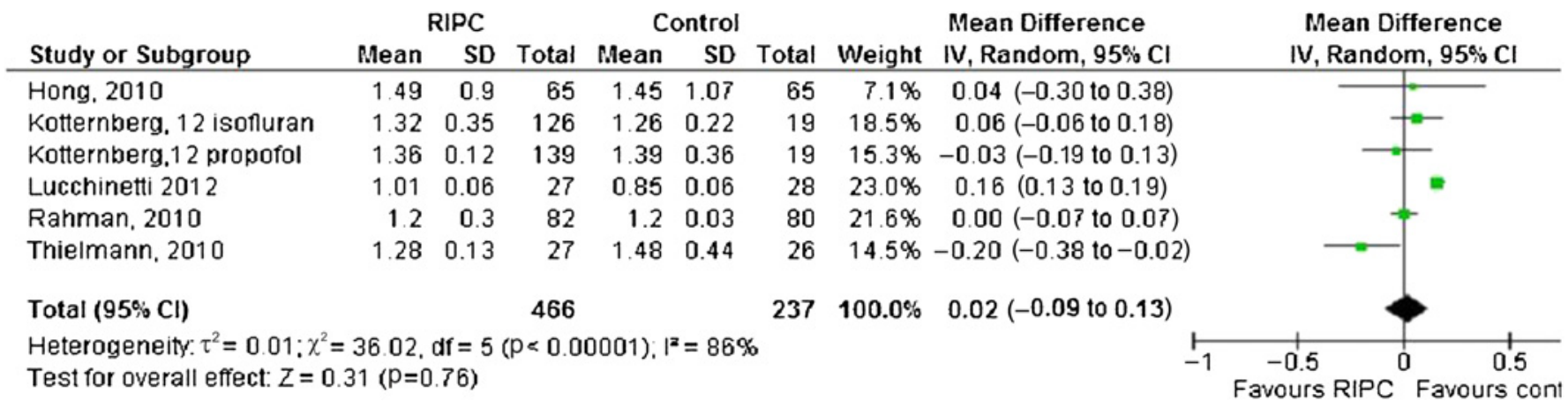

Figure 4 Weighted mean difference of creatinine release $(\mathrm{mg} / \mathrm{dl})$. RIPC, remote ischaemic preconditioning.

burden. Eventually, RIPC showed a systemic anti-inflammatory influence through suppression of pro-inflammatory gene expression activated by the cardiopulmonary bypass circuit and the ischaemia-reperfusion injury. ${ }^{30}$ The many subcellular events beyond humoural, neural and anti-inflammatory hypothesis are not fully defined. Even though different intracellular signal transduction mechanisms are recruited (such as, reactive oxygen species, nitric oxide, and the mitochondrial KATP channel), the ATP-sensitive potassium channel on the inner mitochondrial membrane appears to be the critical one in promoting cell survival. ${ }^{30} 31$

In the present study, the protective effect of RIPC on troponin release was consistently independent from anaesthetic and surgical technique. Continued evolution of cardioprotective techniques, recognition that some anaesthetics can induce cardioprotection, and changes in surgical methods have confounded the translation of these results to modern practice. ${ }^{31}$ Some preliminary considerations around the RCTs included are useful for interpretation of present results; the protocol employed to administer remote upper arm preconditioning showed substantial reproducibility in almost all trials. Isofluorane, as anaesthetic, ${ }^{2425}$ and cross-clamp fibrillation, ${ }^{32-34}$ although with different pathways, were demonstrated to enhance a preconditioning protection in animal models with a reduction in infarct size, thus questioning the positive effect of RIPC in patients treated with these strategies. In the present analysis, the troponin release was reduced in patients undergoing pre-conditioning after the inclusion or not of RCTs with cross-clamp fibrillation and isofluorane as surgical and anaesthetic technique, respectively, stressing its value in different operative settings.

However, these findings should be regarded with caution since no clinical end-point was taken into account in the vast majority of studies, and despite being limited by small sample size, no reduction in length of in-hospital stay was noted. Myocardial injury remains an important predictor of adverse outcome, and the secondary inflammatory process contributes to postoperative morbidity. ${ }^{4}$ To our knowledge, a randomised clinical trial $^{35}$ is recruiting patients to test the hypothesis that the reduced troponin release demonstrated from our analysis will translate into clinical benefits.

Although the bulk of research focuses on the heart as the preconditioned organ, RIPC systemic anti-inflammatory influence proved to be protective against renal ischaemia-reperfusion injury. ${ }^{3} 11$ Interestingly, our data do not support these previous evidences. Possible explanations may be the exclusion criteria of patients with renal disease from the original studies. These populations would be at higher risk for postoperative kidney injury, and therefore, may derive greater benefit from RIPC. Other possible interfering factors could have been the intravenous fluid administration during the postoperative period (which carries a potential reduction of creatinine serum concentrations), and the variation of glomerular filtration rate caused by instability of perfusion rather than kidney damage. Unfortunately, the original studies did not provide correction for these potential biases.

The present article shares many limitations. First, all RCTs consisted of a small sample size, a common feature of surgical work, ${ }^{36}$ thus not allowing us to be conclusive, but stimulating enough to trigger larger trial designs. ${ }^{35}$ Anyway, their quality was acceptable according to international criteria, and no smallstudy bias was assessed (online supplementary figures A and B). We pooled all troponin values together according to smd, which, as suggested from Cochrane, represents a useful tool to compare the same clinical outcome measured with different scales. Another limit is that none of the included studies was powered to detect clinical differences evaluated in our analysis. Heterogeneity was in most cases mild; however, more conservative random effects were exploited. The protocols were similar, although one trial was with off-pump CABG and one with fibrillation. Moreover, similar trends were found after exclusion of studies with different surgical or anaesthetic protocols.

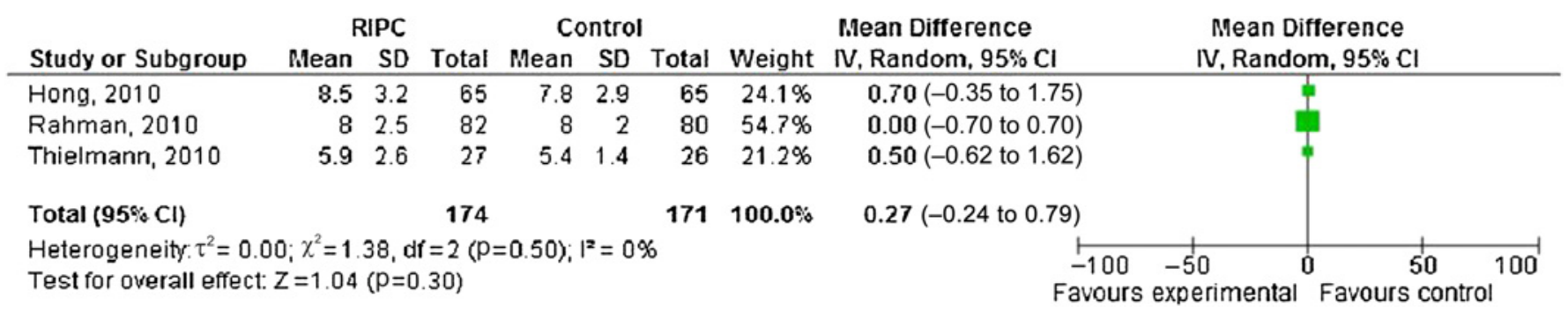

Figure 5 Weighted mean difference of in-hospital stay (days). RIPC, remote ischaemic preconditioning. 
In summary, we found that RIPC reduced troponin release in patients undergoing CAGB. A surrogate clinical end-point (length of in-hospital stay) and renal protection did not demonstrate a significant decrease. Further research exploring RIPC is needed to investigate whether the reduction of myocardial injury observed impacts on clinical outcomes, and to test renal protection through biomarkers insensitive to haemodynamic effects.

Contributors $\mathrm{FDA}, \mathrm{EC}, \mathrm{CM}$, and $\mathrm{GBZ}$ conceived the paper, and wrote it along with $\mathrm{PO}$, FS, MGM, IS and FG. IAR, RSB, YMA, RW, TF, GK, MSM, MT, BJ and YMA gave data from their studies and participated in the drawing up of the discussion.

Competing interests None.

Patient consent Obtained.

Provenance and peer review Not commissioned; internally peer reviewed.

\section{REFERENCES}

1. Przyklenk K, Bauer B, Ovize M, et al. Regional ischemic 'preconditioning' protects remote virgin myocardium from subsequent sustained coronary occlusion. Circulation 1993;87:893-9.

2. Cheung MM, Kharbanda RK, Konstantinov IE, et al. Randomised controlled trial of the effects of remote ischemic preconditioning on children undergoing cardiac surgery: first clinical application in humans. J Am Coll Cardiol 2006:47:2277-82.

3. Ali ZA, Callaghan CJ, Lim E, et al. Remote ischemic preconditioning reduces myocardial and renal injury after elective abdominal aortic aneurysm repair: a randomized controlled trial. Circulation 2007:116:198-105.

4. Hoole SP, Heck PM, Sharples L, et al. Cardiac remote ischaemic preconditioning in coronary stenting (CRISP Stent) Study: a prospective, randomized control trial. Circulation 2009:119:820-7.

5. Hausenloy DJ, Mwamure PK, Venugopal V, et al. Effect of remote ischaemic preconditioning on myocardial injury in patients undergoing coronary artery bypass graft surgery: a randomised controlled trial. Lancet 2007;370:575-9.

6. Croal BL, Hillis GS, Gibson PH, et al. Relationship between postoperative cardiac troponin I levels and outcome of cardiac surgery. Circulation 2006:114:1468-75.

7. Fellahi JL, Gue X, Richomme $X$, et al. Short- and long-term prognostic value of postoperative cardiac troponin I concentration in patients undergoing coronary artery bypass grafting. Anesthesiology 2003;99:270-4.

8. Rahman IA, Mascaro JG, Steeds RP, et al. Remote ischemic preconditioning in human coronary artery bypass surgery: from promise to disappointment? Circulation 2010;122(11 Suppl):S53-9.

9. Wilczynski NL, Haynes RB; for the Hedges Team. Developing optimal search strategies for detecting clinically sound prognostic studies in MEDLINE: an analytic survey. BMC Med 2004;2:23.

10. Moher D, Liberati A, Tetzlaff J, et al; PRISMA Group. Preferred reporting items for systematic reviews and meta-analyses: the PRISMA statement. BMJ 2009;339:b2535.

11. Altman DG, Schulz KF, Moher D, et al; CONSORT GROUP (Consolidated Stand Reporting Trials). The revised CONSORT statement reporting randomized trials explanation elaboration. Ann Intern Med 2001:134:663-94.

12. Jadad AR, Moore RA, Carroll D, et al. Assessing the quality of reports of randomized clinical trials: is blinding necessary? Control Clin Trials 1996;17:1-12.

13. Venugopal V, Laing CM, Ludman A, et al. Effect remote ischemic preconditioning acute kidney injury nondiabetic patients undergoing coronary artery bypass graftsurgery a secondary analysis 2 small randomized trials. Am J Kidney Dis 2010:56:1043-9.

14. Amr YM, Yassin IM. Cardiac protection during on-pump coronary artery bypass grafting: ischemic versus isoflurane preconditioning. Semin Cardiothorac Vasc Anesth 2010;14:205-11.

15. Wu ZK, Laurikka J, Saraste A, et al. Cardiomyocyte apoptosis and ischemic preconditioning in open heart operations. Ann Thorac Surg 2003;76:528-34.
16. Ji B, Liu M, Liu J, et al. Evaluation by cardiac troponin I: the effect of ischemic preconditioning as an adjunct to intermittent blood cardioplegia on coronary artery bypass grafting. J Card Surg 2007;22:394-400.

17. Ali N, Rizwi F, lqbal A, et al. Induced remote ischemic pre-conditioning on ischemiareperfusion injury in patients undergoing coronary artery bypass. J Coll Physicians Surg Pak 2010;20:427-31.

18. Wagner R, Piler $\mathrm{P}$, Bedanova $\mathrm{H}$, et al. Myocardial injury is decreased by late remote ischaemic preconditioning and aggravated by tramadol in patients undergoing cardiacsurgery: a randomised controlled trial. Interact Cardiovasc Thorac Surg 2010;11:758-62

19. Venugopal V, Hausenloy DJ, Ludman A, et al. Remote ischaemic preconditioning reduces myocardial injury in patients undergoing cardiac surgery with cold-blood cardioplegia: arandomised controlled trial. Heart 2009;95:1567-71.

20. Hausenloy DJ, Mwamure PK, Venugopal V, et al. Effect of remote ischaemic preconditioning on myocardial injury in patients undergoing coronary artery bypass graft surgery: arandomised controlled trial. Lancet 2007;370:575-9.

21. Hong DM, Mint JJ, Kim JH, et al. The effect of remote ischaemic preconditioning on myocardial injury in patients undergoing off-pump coronary artery bypass graft surgery. Anaesth Intensive Care 2010;38:924-9.

22. Thielmann M, Kottenberg $\mathrm{E}$, Boengler $\mathrm{K}$, et al. Remote ischemic preconditioning reduces myocardial injury after coronary artery bypass surgery with crystalloid cardioplegic arrest. Basic Res Cardiol 2010;105:657-64.

23. Karuppasamy P, Chaubey S, Dew T, et al. Remote intermittent ischemia before coronary artery bypass graft surgery: a strategy to reduce injury and inflammation? Basic Res Cardiol 2011;106:511-19.

24. Lucchinetti E, Bestmann L, Feng J, et al. Remote ischemic preconditioning applied during isoflurane inhalation provides no benefit to the myocardium of patients undergoing on-pump coronary artery bypass graft surgery: lack of synergy or evidence of antagonism in cardioprotection? Anesthesiology 2012;116:296-310

25. Kottenberg E, Thielmann M, Bergmann L, et al. Protection by remote ischemic preconditioning during coronary artery bypass graft surgery with isoflurane but not propofol—a clinical trial. Acta Anaesthesiol Scand 2012;56:30-8.

26. Gho BC, Schoemaker RG, van den Doel MA, et al. Myocardial protection by brief ischemia in noncardiac tissue. Circulation 1996:94:2193-200.

27. Tang ZL, Dai W, Li YJ, et al. Involvement of capsaicin-sensitive sensory nerves in early and delayed cardioprotection induced by a brief ischaemia of the small intestine. Naunyn Schmiedebergs Arch Pharmacol 1999;359:243-7.

28. Konstantinov IE, Li J, Cheung MM, et al. Remote ischemic preconditioning of the recipient reduces myocardial ischemia-reperfusion injury of the denervated donor heart via a Katp channel-dependent mechanism. Transplantation 2005:79:1691-5

29. Battipaglia I, Scalone G, Milo M, et al. Upper arm intermittent ischaemia reduces exercise-related increase of platelet reactivity in patients with obstructive coronary artery disease. Heart 2011;97:1284-5.

30. Konstantinov IE, Arab S, Kharbanda RK, et al. The remote ischemic preconditioning stimulus modifies inflammatory gene expression in humans. Physiol Genomics 2004; 19:143-50.

31. Kharbanda RK. Cardiac conditioning: a review of evolving strategies to reduce ischaemia-reperfusion injury. Heart 2010;96:1179-86.

32. Fujii M, Chambers DJ. Myocardial protection with intermittent cross clamp fibrillation: does preconditioning play a role? Eur J Cardiothorac Surg 2005;28:821-31.

33. Scarci M, Fallouh HB, Young CP, et al. Does intermittent cross-clamp fibrillation provide equivalent myocardial protection compared to cardioplegia in patients undergoingbypass graft. Interact Cardiovasc Thorac Surg 2009;9:872-8.

34. Rerkasem K, Rothwell PM. Meta-analysis small randomized controlled trials surgery may be unreliable. Br J Surg 2010;97:466-9.

35. Hausenloy DJ, Candilio L, Laing $\mathrm{C}$, et al; The ERICCA trial investigators. Effect of remote ischemic preconditioning on clinical outcomes in patients undergoing coronary artery bypass graft surgery (ERICCA): rationale and study design of a multicentre randomized double-blinded controlled clinical trial. Clin Res Cardiol. Published Online First: 21 December 2011.

36. Yusuf S. Meta-analysis of randomized trials: looking back and looking ahead. Control Clin Trials 1997:18:594-601; discussion 661-6. 


\section{Heart}

Remote ischaemic preconditioning in coronary artery bypass surgery: a meta-analysis

Fabrizio D'Ascenzo, Erika Cavallero, Claudio Moretti, Pierluigi Omedè, Filippo Sciuto, Ishtiaq A Rahman, Robert S Bonser, Jeon Yunseok, Robert Wagner, Tomas Freiberger, Gudrun Kunst, Michael S Marber, Matthias Thielmann, Bingyang Ji, Yasser M Amr, Maria Grazia Modena, Giuseppe Biondi Zoccai, Imad Sheiban and Fiorenzo Gaita

Heart 2012 98: 1267-1271

doi: 10.1136/heartjnl-2011-301551

Updated information and services can be found at:

http://heart.bmj.com/content/98/17/1267

These include:

References This article cites 35 articles, 10 of which you can access for free at: http://heart.bmj.com/content/98/17/1267\#BIBL

Email alerting Receive free email alerts when new articles cite this article. Sign up in the service box at the top right corner of the online article.

Topic Articles on similar topics can be found in the following collections

Collections

Interventional cardiology (2932)

\section{Notes}

To request permissions go to:

http://group.bmj.com/group/rights-licensing/permissions

To order reprints go to:

http://journals.bmj.com/cgi/reprintform

To subscribe to BMJ go to:

http://group.bmj.com/subscribe/ 